\title{
Relativistic Thermodynamics and Kinetics of Electrode Processes
}

\author{
Mirza Wasif Baig ${ }^{1}$ \\ ${ }^{1}$ J. Heyrovsky Institute of Physical Chemistry, Academy of Sciences of the Czech Republic, \\ CZ-18223 Prague 8, Czech Republic \\ wasifbaig.mirza@jh-inst.cas.cz
}

\begin{abstract}
From the theory of relativistic chemical kinetics [M. W. Baig, Int. J. Mod. Phys. B 31, 1750177 (2017)] relativistic thermodynamics and kinetics for electrode processes have been developed to explain time dilation for electrode processes. For a moving observer moving at fractions of the speed of light, cell potential is observed to decrease. This results in the slower oxidation and reduction of ions at the respective electrodes. The newly formulated Lorentz transformation of the electrode and cell potential is explained in terms of generation of spin 2-boson "gravitons" from fusion of spin-1 boson "virtualphotons" mediating electrostatic force of attraction between ions and electrodes. It is postulated that birth of spin 2-boson i.e. gravitons is followed by their eventual escape in any of higher $4+\mathrm{n}$ dimensions. To demonstrate the effectiveness of the present theory, the Daniel cell is considered as a numerical example.
\end{abstract}

PACS numbers: 82.45.Jn, 03.30.+p, 82.60.-s, 82.20.-w, 82.40.-g

\section{Key Words}

relativistic cell potential; relativistic thermodynamics; time dilation; graviton; virtual photon. 


\section{Introduction}

Electrochemistry is an interdisciplinary science which comes from the marriage of electrical physics and chemistry. $\cdot$ Majorly in electrochemistry redox reactions (oxidation and reduction) occurring at the interface of electrode and electrolyte are studied which is occurs in liquid phase. Electrochemical reactions occurring at surface of electrode can be broadly classified in two categories non spontaneous and spontaneous redox reactions. Non spontaneous redox reactions are carried out by passing electric current through the cell by applying at least threshold potential across the electrodes of the cell while spontaneous redox reactions governs on their own when electrodes are brought in contact with suitable electrolyte which generate potential difference across the electrodes that lead to flow of electric current through the external circuit. ${ }^{2}$ Recently relativistic thermodynamics and kinetics have been formulated to explain time dilation for chemical reactions in a moving frame of reference. ${ }^{3}$ Time dilation is one of the basic foundations of special theory of relativity. ${ }^{4}$ In relativistic theory of chemical kinetics Lorentz transformation of rate constant and thermodynamic state functions have been formulated which are compatible with time dilation phenomenon, which is an experimentally verified fact. ${ }^{5}$ The present paper is an attempt to formulate Lorentz transformations that can explain time dilation in the kinetics of electrochemical phenomenon. In present paper all the variables with subscript $o$ refer to the inertial frame of reference $K_{o}$ with the observer at rest while variables with subscript $u$ refer to the inertial frame $K_{u}$ with the observer moving at relative speed $u$. All Lorentz transformations are defined in terms of a factor " $\gamma=$ $\left(1-u^{2} / c^{2}\right)^{-1 / 2} "$

\section{Theory}

To formulate theory that can explain time dilation for electrochemical process from an eye of moving observer, let consider following reaction occurring on the surface of an arbitrary electrode. ${ }^{1}$

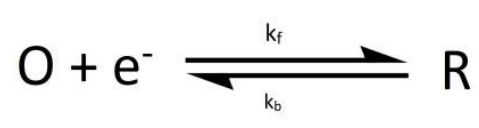

Let in above electrode process specie $\mathrm{O}$ and $\mathrm{R}$ is being involved in one electron transfer process. Free energy associated with above one electron electrode process for moving observer can be formulated as, $\underline{6}$

$$
\Delta G_{u}=-n F E_{u}
$$

As free energy is proved to be Lorentz variant i.e. $\Delta G_{u}=\gamma^{-1} \Delta G_{o},{ }^{3}$ it is observed to be less for moving observer. Since according to special theory of relativity laws of physics holds good in all inertial frames. So to hold this basic postulate of special relativity electrode potential becomes Lorentz variant i.e.

$$
E_{u}=\gamma^{-1} E_{o}
$$

Rate constants of forward and backward reaction cab be formulated in terms of Arrhenius factor and free energy of activation as, ${ }^{7-8}$ 


$$
\begin{aligned}
& \left(k_{f}\right)_{u}=\left(A_{f}\right)_{u} \exp \left(-\left(\Delta G_{0 a}^{\dagger}\right)_{u} / R_{u} T\right) \exp \left[-\alpha\left(E_{u}-E_{u}^{0} / R_{u} T\right)\right. \\
& \left(k_{b}\right)_{u}=\left(A_{b}\right)_{u} \exp \left(-\left(\Delta G_{0 b}^{\dagger}\right)_{u} / R_{u} T\right) \exp \left[(1-\alpha)\left(E_{u}-E_{u}^{0} / R_{u} T\right)\right.
\end{aligned}
$$

For a special case $E_{u}=E_{u}^{0}$ rate constant for forward and backward reaction become equal i.e.

$$
\left(k_{0}\right)_{u}=\left(A_{b}\right)_{u} \exp \left(-\left(\Delta G_{0 b}^{\dagger}\right)_{u} / R_{u} T\right)=\left(A_{f}\right)_{u} \exp \left(-\left(\Delta G_{0 a}^{\dagger}\right)_{u} / R_{u} T\right)
$$

On substitution of Lorentz transformation of Arrhenius factor, ideal gas constant free activation energy in Eq. (5) gives same Lorentz transformation of threshold rate constant i.e. $\left(k_{0}\right)_{u}=\gamma^{-1}\left(k_{0}\right)_{o}$. Eq.s (3) and (4) can be written in more contact form as, $\stackrel{1-2}{ }$

$$
\begin{aligned}
& \left(k_{f}\right)_{u}=\left(k_{0}\right)_{u} \exp \left[-\alpha\left(E_{u}-E_{u}^{0} / R_{u} T\right)\right. \\
& \left(k_{b}\right)_{u}=\left(k_{0}\right)_{u} \exp \left[(1-\alpha)\left(E_{u}-E_{u}^{0} / R_{u} T\right)\right.
\end{aligned}
$$

On substitution of Lorentz transformation of Electrode potential, ideal gas constant and threshold rate constant in Eq.s (6) and (7) gives same Lorentz transformation of rate constant as formulated earlier, ${ }^{3}$

$$
\begin{aligned}
& \left(k_{f}\right)_{u}=\gamma^{-1}\left(k_{f}\right)_{o} \\
& \left(k_{b}\right)_{u}=\gamma^{-1}\left(k_{b}\right)_{o}
\end{aligned}
$$

Butler-Volmer relation that explains total current flow in an electrode process for moving observer can be stated as,

$$
i_{u}=N F A\left(k_{0}\right)_{u}\left[\operatorname { e x p } \left[-\alpha\left(E_{u}-E_{u}^{0} / R_{u} T\right)-\exp \left[(1-\alpha)\left(E_{u}-E_{u}^{0} / R_{u} T\right)\right]\right.\right.
$$

On substitution of Lorentz transformation of Electrode potential, ideal gas constant and threshold rate constant in Eq.s (10) gives Lorentz transformation of current as, ${ }^{3}$

$$
i_{u}=\gamma^{-1} i_{o}
$$

So due to drop in electric potential for moving observer net current flow will slow down. Moving observer will find net charge transfer per unit time less than compare to in its own frame of reference because for moving observer clock of system at rest will ticks slower.

\section{Discussion}

Migration of ions towards their respective electrodes is due to electrostatic force of attraction between the electrode and electrolyte. Potential applied externally on the electrode or potential developed on the electrode when it comes in contact with the electrolyte makes it either positively or negatively charged which attract the ions of opposite charge towards itself to go under respective redox reaction. Now according to Eq. (2) when cell potential will be observed to decrease for moving observer then it means electrostatic force of attraction between ions and electrode will fall in magnitude for moving observer. To explain this fact we have to take in account some concepts from particle physics. ${ }^{9}$ For every force existing in nature there is a particle that mediates corresponding natural force. ${ }^{10}$ For electrostatic force of attraction 
this messenger particle is photon which is a boson with spin 1. ${ }^{11-12}$ In present paper decrease in potential for moving observer is explained in terms of interconversion of virtual photons in to gravitons. For gravitational force of attraction messenger particle is a spin 2-boson named as graviton which can escape in to any of the possible higher dimensions. ${ }^{13-14}$ Gravitation is explained as a weak force by unique feature of gravitons i.e. they possess closed strings resulting in leakage of gravitons from the brane into higherdimensional space. ${ }^{15-16}$ Escaping of gravitons in to any of higher $4+n$ dimensions is now a well known and well established theoretical concept very commonly found in literature. ${ }^{17-18} \mathrm{D}$. Boccaletti and et al. have explained production of gravitons from either fusion of photons in presence of either gravitational field or electromagnetic field. ${ }^{19-20}$ While production of gravitons during photon-photon, electron-photon and nucleus-nucleus collisions at $\mathrm{TeV}$ scale has already been predicted many times in literature. ${ }^{21-23}$ Potential present between electrode and ions is responsible for electrostatic force attracting ions towards the electrode. In moving frame decrease in potential will result in decrease in magnitude of electromotive force existing between ions and electrode. For moving observer drop in potential and weakening of electromotive force between ions and electrode can be explained by interconversion of virtual photons (mediating electromotive force between ions and electrode) in to gravitons. Potential drop for moving observer at any temperature results from fusion of virtual photons leading to graviton production which eventually escape in to any of higher $4+n$ dimensions. Two virtual photons add up to give a graviton which escape in to higher dimensions. In present theory for the first time it is postulated that for moving observer at any energy scale and temperature two spin-1 boson "photons" can add up to produce a spin 2boson "graviton" which disappears in to any of possible higher $4+\mathrm{n}$ dimensions. This interconversion of photons in to gravitons would result in decrease of potential of cell and electrode for moving observer. So for moving observer some of the photons mediating electrostatic force of attraction between ions and electrode will add up to produce a graviton which is a spin 2-boson which would escape in to any of possible higher $4+\mathrm{n}$ dimensions thus leading to Lorentz transformation of cell and electrode potential for moving observer i.e. $\left(E_{\text {cell/elect }}\right)_{u}=\gamma^{-1}\left(E_{\text {cell/elect }}\right)_{o}$.

\section{Application}

\section{Daniel cell}

Daniel cell is one of the earliest electro voltaic cell in which $\mathrm{Zn}(0)$ is oxidized to $\mathrm{Zn}(\mathrm{II})$ at the anode, $\mathrm{Cu}(\mathrm{II})$ is reduced to $\mathrm{Cu}(0)$ at the cathode, i.e. $\mathrm{Zn}+\mathrm{Cu}^{2+} \rightarrow \mathrm{Zn}^{2+}+\mathrm{Cu}^{6}$ Now, if the moving observer monitors cell potential at speed of $2 \times 10^{8} \mathrm{~ms}^{-1}$ then cell potential will be observed to decrease as shown in the table 1 .

Table 1. Comparison of cell potential for moving and stationary observer at $298 \mathrm{~K}$

\begin{tabular}{|c|c|}
\hline Net cell potential for stationary observer (V) & Net cell potential for stationary observer (V) \\
\hline 1.10 & $0.819^{\mathrm{a}}$ \\
\hline
\end{tabular}

[a] This value has been developed from Eq. (2) 


\section{Conclusion}

Lorentz transformation of cell potential results in slower rates of forward and backward redox reactions. Moving observer will also find the net current flow to be smaller. In moving frame drop in potential will demand longer time to drive the same charge as that in rest frame of reference; this is totally in agreement with time dilation which explains slower ticking of clocks for moving observer. Decrease in cell potential for moving observer is due to decrease in electromotive force operating between ions and electrodes. This decrease in magnitude of electromotive force and cell potential can be theoretically explained by fusion of two spin-1 bosons i.e. virtual photons producing spin 2-boson graviton which escape in any of possible $4+\mathrm{n}$ extra dimensions.

\section{References}

1. K. J. Vetter, Electrochemical Kinetics (Academic Press, New York, 1967)

2. Allen J. Bard and Larry R. Faulkner Electrochemical Methods: Fundamentals and Applications (John Wiley and Sons. Inc. New York $20012^{\text {nd }}$ Edition)

3. M. W. Baig, Int. J. Mod. Phys. B 31, 1750177 (2017).

4. A. Einstein, Annalen der Physik 17, 891(1905).

5. R. E. Hall, D. A. Lind and R. A. Ristinen, Am. J. Phys. 38, 1196 (1970).

6. T. Engel and P. Reid, Thermodynamics, Statistical Thermodynamics and Kinetics, (Prentice Hall, Harlow $20063^{\text {rd }}$ Edition)

7. J.A.V Butler, Trans. Farad. Soc. 19, 729 (1924).

8. T. Erdey-Gruz and M. Volmer, Z. Physik. Chem. 150A, 203 (1930).

9. J. Jaeckel and A. Ringwald, Annu. Rev. Nucl. Part. Sci. 60, 405 (2010)

10. K. Huang, Fundamental Forces of Nature The Story of Gauge Fields (World Scientific Publishing Co. Pte. Ltd. Singapore 2007)

11. J.H. Field Phys. Scr. 74, 702 (2006)

12. M. Conrad Physics Letters A 152, 245 (1991).

13. M.Sher and K. A. Sullivan, Am. J. Phys. 74, 145 (2006).

14. V. H. S. Kumar, P. K. Suresh and P. K. Das, AIP Conference Proceedings 939, 258 (2007).

15. I. Antoniadis, N. A. Hamed, S. Dimopoulos and G. Dvali Physics Letters B, 436, 257 (1998).

16. H. Murayama and J. D. Wells, Phys Rev. D, 65, 056011 (2002) 
17. N. A. Hamed, S. Dimopoulos and G. Dvali, Physics Letters B 429, 263 (1998).

18. S. Creek, O. Efthimiou, P. Kanti and K. Tamvakis Physics Letters B 635, 39 (2006).

19. D. Boccaletti, V. De Sabbata, C. Gualdi and P. Fortini IL Nuovo Cimento 54, 134 (1968)

20. D. Boccaletti, V. De Sabbata, C. Gualdi and P. Fortini IL Nuovo Cimento 70, 129 (1970)

21. D. Atwood, S. B. Shalom and A. Soni, Phys. Rev. D 61, 116011 (2000).

22. S.C. Ahern, J. W. Norbury and W. J. Poyser Phys. Rev. D 62, 116011 (2000).

23. P. de Aquino, K. Hagiwara, Q. Li and F. Maltoni, JHEP 6, 132 (2011). 